\title{
Os custos da inatividade física no mundo: estudo de revisão
}

\author{
The costs of physical inactivity in the world: a general review
}

Denise Rodrigues Bueno ${ }^{1}$

Maria de Fátima Nunes Marucci ${ }^{1}$

Jamile Sanches Codogno ${ }^{2}$

Manuela de Almeida Roediger ${ }^{1}$

${ }^{1}$ Pós-Graduação em Nutrição em Saúde Pública, Faculdade de Saúde Pública,Universidade de São Paulo. Av. Dr. Arnaldo 715, Cerqueira César. 01246904 São Paulo SP Brasil. denibueno@hotmail.com ${ }^{2}$ Faculdade de Ciências e Tecnologia de Presidente Prudente, Universidade Estadual Paulista Júlio de Mesquita Filho. São Paulo SP Brasil.

\begin{abstract}
There is convincing evidence in the scientific literature of the effectiveness of regular physical activity and physical exercise in the conservation of health and the prevention of various ailments. However, studies into the association between costs of medical services and physical inactivity have not been duly addressed. International studies have quantified these costs and revealed the association between physical activity and/or sedentary behavior. Therefore, this review sought to gather information available from several countries and analyze the global costs associated with physical inactivity over the past few decades. The results of twenty-four original and well-researched articles in nine countries, including Brazil, were analyzed. The results showed that physical inactivity, irrespective of the method of classification, is burdensome to the economy of health worldwide, and directly responsible for the high cost of medication, the incidence of hospitalization and the frequency of medical appointments. The costs of the group of the physically inactive population affected by chronic diseases feature among the major components of the total costs involved in public health.
\end{abstract}

Key words Review, Physical activity, Financial resources in health
Resumo Os beneficios da atividade fisica e do exercício físico para a manutenção da saúde e a prevenção de doenças estão bem esclarecidos na literatura científica. Entretanto, estudos associando os custos da utilização de serviços de saúde e com os da inatividade física ainda são poucos. Pesquisas internacionais quantificaram estes custos e apresentaram associações com a prática de atividade fisica e/ou comportamento sedentário. Assim, o objetivo desta revisão foi, a partir destas informações, analisar os custos mundiais relacionados à inatividade física nas últimas décadas. Foram utilizados os resultados de 24 artigos originais, conduzidos em nove países, incluindo o Brasil. Os resultados mostraram que a inatividade física, independente do método de classificação, é onerosa à economia da saúde em todo o mundo $e$ diretamente responsável pelo alto gasto com medicamentos, internação hospitalar e consultas clínicas. Os gastos com a parcela da população fisicamente inativa, acometida por doenças crônicas,estão entre os principais integrantes dos custos totais em saúde pública.

Palavras-chave Revisão, Atividade física, Recursos financeiros em saúde 


\section{Introdução}

Estima-se que 6\% das doenças cardiovasculares e $7 \%$ dos casos de diabete tipo 2 (DM2) no mundo são causados por inatividade física ${ }^{1}$. O impacto dessa relação sobre os custos com saúde em países desenvolvidos é preocupante, uma vez que até $3 \%$ dos recursos financeiros disponíveis sejam utilizados devido à inatividade física² ${ }^{2}$ Em 2012, os custos diretos contabilizados devidos à DM2 no Reino Unido chegaram a 20 bilhões de dólares (USD\$) ${ }^{3}$, e na Escócia, estimativas apontam que a redução de $1 \%$ de inativos a cada ano significaria a economia de USD\$ 130 milhões, devido à prevenção de mortalidade por doença cardíaca ${ }^{4}$.

Em 2012, 10,1\% do Produto Interno Bruto (PIB) em todo o mundo foram gastos com recursos para a saúde. No Brasil, este percentual é de $9 \%$ ao ano, o qual se assemelha ao do Japão, Itália e Austrália 5 . A análise econômica em saúde é utilizada para a tomada de decisões em relação à alocação de recursos, tanto em âmbito de saúde pública, quanto privada. As doenças crônicas representam $60 \%$ do total de doenças no país ${ }^{6}$ e, embora o efeito do exercício e da atividade física (AF) para a manutenção da saúde e controle de doenças esteja bem estabelecido na literatura ${ }^{7}$, a prevalência de sedentarismo no tempo livre é alta $^{8}$ e independente do grupo etário, atingindo $80 \%$ dos idosos ${ }^{9}$.

Estudos internacionais a respeito dessa temática são mais numerosos e fornecem dimensões da associação entre utilização de recursos e nível de atividade física (NAF) em diferentes países. No Brasil, alguns estudos analisaram os custos relacionados à inatividade física, em nível populacional, especialmente sobre os recursos públicos. Assim, o objetivo desta pesquisa foi compilar informações destes estudos sobre os custos com saúde decorrentes da inatividade física, visando maiores esclarecimentos da utilização de recursos com saúde no mundo.

\section{Métodos}

Os artigos foram localizados pelas bases de dados PubMed-Medline, Bireme e SciELO, em fevereiro de 2014, utilizando os seguintes conjuntos de palavras-chave: "physicalactivity + costs + healthcare"; "physicalinactivity + costs + healthcare"; "physicalinactivity + costs"; "atividade física + custos" e "inatividade física + custos".

Os dados apresentados na Figura 1 mostram as quantidades de artigos encontrados nas bases de dados, a partir dos termos mencionados anteriormente.

Foram considerados para a presente pesquisa artigos em língua portuguesa, inglesa ou espanhola, publicadas a partir de 1990. Para inclusão nas análises, os artigos deveriam ser originais e explorar, especificamente, o tema da AF e sua relação com os custos diretos com a utilização de serviços de saúde por adultos e idosos, tais comoconsultas clínicas, hospitalizações, serviço de emergência, medicamentos, planos de saúde, entre outros. Também foram explorados os trabalhos cujo tema abordasse a análise de custo-efetividade da intervenção com programas de atividade física.

A leitura dos abstracts dos artigos selecionados por meio das palavras-chave permitiu a exclusão daqueles que não atendiam a todos os critérios propostos: i) os artigos de revisão, incluindo as meta-análises; ii) as pesquisas realizadas com população adolescente, iii) pesquisas que tratassem de custos entretanto, sem abordar a sua relação com o NAF; e, iv) artigos publicados antes de 1990.

Após a seleção dos trabalhos considerados adequados pelos autores por meio da leitura dos abstracts, foram analisados os resultados de 24 artigos oriundos de pesquisas, desenvolvidas em nove países: Austrália, Brasil, Canadá, China, Estados Unidos (EUA), Itália Inglaterra, Japão e Suécia. Para facilitar interpretação e a comparação dos resultados entre os estudos, os valores de custos de todos os países foram convertidos para Dólares Americanos (USD\$), considerando a cotação no período de análise de cada pesquisa.

\section{Resultados}

Das 24 pesquisas, oito realizaram estimativas populacionais, considerando os custos referentes ao hábito sedentário a partir da sua associação com a prevalência de doenças crônicas ${ }^{10-17}$. Cinco se tratam de estudos de coorte, com seguimento médio de dois anos ${ }^{18-22}$. E o restante, refere-se a estudos transversais, entre os quais existem publicações com populações específicas compostas de pacientes diabéticos ${ }^{21,23-25}$, idosos ${ }^{26-29}$ e mulheres ${ }^{20,30}$.

As análises dos resultados mostraram que quanto maior o NAF menor o uso e custos com de medicamentos ${ }^{21,23,27,31}$, consultas clínicas ${ }^{24}$ e hospitalizações ${ }^{29,32}$. Mesmo quando consideradas as atividades não sistematizadas, como a recreação, a atividade doméstica e a ocupacional, houve relação inversa entre o gasto energético dispendi- 
Figura 1. Número de artigos localizados em cada base de dados, de acordo com as palavras-chave.

do (kcal) nestas tarefas com os custos em saúde $(\mathrm{r}=-0,22 ; \mathrm{p}<0,01)^{28}$.

O Quadro 1 apresenta o resumo dos resultados que destacaram a comparação feita, pelos autores, entre as variáveis de custo e NAF. É possível observar que todos os artigos analisados indicam custos superiores para pessoas inativas, quando comparadas às pessoas fisicamente ativas. Três dos estudos analisaram os custos de pessoas com 65 anos ou mais e mostraram custos maiores em fisicamente inativos.

Nos Estados Unidos, embora analisando o NAF de maneiras distintas, os estudos mostram maiores custos anuais com saúde em indivíduos classificados como fisicamente inativos ${ }^{26,27}$, principalmente em relação às hospitalizações ${ }^{32}$. Os custos atribuídos à ausência de AF no tempo livre no ano 2000 foram de USD\$ 10,8 milhões, para tratamento de hipertensão arterial sistêmica (HAS), e de USD\$ 7,2 milhões, para diabete. Além disso, ao menos um terço dos custos com doença cardíaca, câncer de cólon e osteoporose foram relacionados à inatividade física ${ }^{17}$.

$\mathrm{Na}$ Austrália, Brown et al. ${ }^{30}$ analisaram os dados do The Australian Longitudinal StudyonWomens Health (ALSWH), de uma amostra composta por 7004 mulheres com idade entre 50 e 55 anos. Observou-se que as obesas e inativas apresentaram custo anual $43,2 \%$ maior, do que aquelas com o NAF moderado e IMC $<25 \mathrm{~kg} /$ $\mathrm{m}^{2}$. No grupo das mulheres com IMC $<25 \mathrm{~kg} /$ $\mathrm{m}^{2}$, o custo foi maior quanto menor o NAF, com o custo de USD\$ 246 em mulheres com NAF moderado e de USD\$320 com NAF insuficiente (< $40 \mathrm{MET} / \mathrm{minuto/semana).}$

Dados derivados de um estudo prospectivo observacional no Japão ${ }^{33}$, no período entre 2002 e 2008, com uma amostra de 483 pessoas com média de idade de 75,5 anos foram analisados por Yang et al. ${ }^{33}$. A prática de esportes foi considera- da como a atividade mais intensa, o NAF baixo foi considerado, quando da ausência de pratica esportiva e de caminhada e, por fim, a atividade moderada foi atribuída aos relatos de caminhada rápida. Também foram analisados testes de potência muscular, força e resistência para análise da aptidão física. O modelo de regressão desse estudo mostrou que, quanto maior o NAF menor é o custo $(\mathrm{p}=0,02)$ e, custos menores para o grupo com melhor performance nos testes físicos $(\mathrm{p}=0,01)$.

Entre os estudos de estimativas populacionais, a partir dos resultados apresentados no Quadro 2, é possível observar que, de forma geral, as pesquisas mostram que as reduções nas prevalências de inatividade física e/ou sedentarismo resultariam diretamente em redução nos custos com saúde. Em relação aos custos totais populacionais dispendidos com saúde, os atribuídos à inatividade física consumiram entre 2,5\% dos recursos disponíveis no Canadá ${ }^{12}$ e $46 \%$ na Inglaterra $^{15}$.

No Brasil, haveria economia de USD\$ 1,14 bilhões dos recursos em saúde devido ao menor número de internações por DM2 e pelo menor uso de medicamentos para diabetes e hipertensãocaso a prevalência de sedentarismo fosse $50 \%$ menor que a atual ${ }^{10}$. No ano 2000, no Canadá, foi estimado que a redução de $10 \%$ no número de indivíduos sedentários na população (GE < $12,6 \mathrm{~kJ} / \mathrm{kg}$ de peso corporal por dia) significaria a economia de USD\$ 150 milhões por ano nos custos diretos com saúde ${ }^{12}$. Em 2009, os custos diretos, indiretos e totais atribuídos à inatividade física ( $<150$ minutos por semana de AF moderada e vigorosa) somaram USD\$2,09, USD\$ 3,74 e USD \$ 5,9 bilhões, respectivamente. Estes valores representaram 3,8\%, 3,6\% e 3,7\% dos custos totais com os recursos de saúde no ano ${ }^{13}$.

$\mathrm{Na}$ Inglaterra, pesquisadores estimaram que 9\% dos recursos para a saúde foram consumidos 
Quadro 1. Descrição dos resultados dos estudos sobre custos diretos com saúde entre diferentes níveis de atividade física (NAF).

\begin{tabular}{|c|c|c|c|c|c|}
\hline \multirow[t]{2}{*}{ Autor } & \multirow{2}{*}{$\begin{array}{l}\text { Ano do } \\
\text { estudo }\end{array}$} & \multirow{2}{*}{$\begin{array}{l}\text { Grupos } \\
\text { etários }\end{array}$} & \multicolumn{2}{|c|}{ Custos } & \multirow{2}{*}{ Classificação do NAF } \\
\hline & & & Ativos & Inativos & \\
\hline Wang et al. ${ }^{26}$ & 2004 & $\begin{array}{l}<65 \text { anos } \\
\geq 65 \text { anos }\end{array}$ & $\begin{array}{l}\text { USD } \$ 3784 \\
\text { USD } \$ 4796\end{array}$ & $\begin{array}{l}\text { USD\$ } 6313 \\
\text { USD\$ } 7425\end{array}$ & $\begin{array}{l}\text { Ativo: } \geq 150 \text { minutos/semana em } \\
\text { atividades moderadas }\end{array}$ \\
\hline Wang et al. ${ }^{27}$ & 2005 & $\geq 65$ anos & USD\$ 9436 & USD\$ 12795 & $\begin{array}{l}\text { Sedentário: não realiza atividade na } \\
\text { semana; Moderadamente ativo: } 1 \text { a } 3 \text { dias } \\
\text { por semana, } 20 \text { minutos por dia; } \\
\text { Muito ativo: } \geq 4 \text { dias/semana }\end{array}$ \\
\hline $\begin{array}{l}\text { Woolcot et } \\
\text { al. }{ }^{29}\end{array}$ & 2010 & $\begin{array}{l}<65 \text { anos } \\
\geq 65 \text { anos }\end{array}$ & $\begin{array}{l}\text { Custos totais } \\
\text { USD\$ } 743 \\
\text { Custos } \\
\text { internação } \\
\text { USD } 436,00\end{array}$ & $\begin{array}{l}\text { Custos totais } \\
\text { USD\$ } 1884 \\
\text { Custos } \\
\text { internação } \\
\text { USD\$ } 1687\end{array}$ & $\begin{array}{l}\text { Ativo: dispêndio de energia em } \mathrm{AF} \geq 1000 \\
\mathrm{kcal} / \mathrm{semana}\end{array}$ \\
\hline $\begin{array}{l}\text { Brown et } \\
\text { al. }^{30}\end{array}$ & 2008 & $\begin{array}{l}\text { Mulheres } \\
50 \text { a } 55 \\
\text { anos }\end{array}$ & $\begin{array}{l}\geq 1200 \mathrm{METs} \\
\text { USD\$ } 289\end{array}$ & $\begin{array}{l}<40 M E T \\
\text { USD } \$ 361\end{array}$ & $\begin{array}{l}\text { Inativas: }<40 \text { METs por minuto/semana } \\
\text { em atividades moderadas e vigorosas; } \\
\text { Moderadamente ativas: } 600 \text { a } 1200 \mathrm{METs} \\
\text { por minuto/semana; } \\
\text { NAF alto: } \geq 1200 \mathrm{METs} \text { por minuto/ } \\
\text { semana }\end{array}$ \\
\hline Yang et al. ${ }^{33}$ & 2011 & $\begin{array}{l}\quad 483 \\
\text { indivíduos } \\
\geq 70 \text { anos }\end{array}$ & $\begin{array}{l}\text { Custos totais } \\
\text { Baixo NAF } \\
\text { USD\$ 702,0 } \\
\text { Melhor } \\
\text { aptidão física } \\
\text { USD\$ } 724\end{array}$ & $\begin{array}{l}\text { Custos totais } \\
\text { Alto NAF } \\
\text { USD } \$ 827,3 \\
\text { Pior aptidão } \\
\text { física } \\
\text { USD } \$ 898\end{array}$ & $\begin{array}{l}\text { Prática regular de esportes (autorreferida) } \\
\text { Performance em testes de aptidão física } \\
\text { (força muscular, teste de caminhada, } \\
\text { flexibilidade, agilidade) }\end{array}$ \\
\hline $\begin{array}{l}\text { Anderson et } \\
\text { al. }{ }^{19}\end{array}$ & 2005 & $\begin{array}{l}\text { Indivíduos } \\
\geq 40 \text { anos }\end{array}$ & USD\$ 3994 & USD\$ 5783 & $\begin{array}{l}\text { Inativos: prática de } \mathrm{AF}=0 \mathrm{dia} / \mathrm{semana} \\
\text { Ativos: prática de } \mathrm{AF} \geq 4 \text { dias/semana de } \mathrm{AF}\end{array}$ \\
\hline $\begin{array}{l}\text { Perkins e } \\
\text { Clark }^{22}\end{array}$ & 2001 & $\begin{array}{c}655 \text { pessoas } \\
\geq 55 \text { anos }\end{array}$ & $\begin{array}{l}\text { Serviço de } \\
\text { emergência } \\
\text { USD } \$ 253 \\
\text { Custos totais } \\
\text { USD\$ } 1865\end{array}$ & $\begin{array}{l}\text { Serviço de } \\
\text { emergência } \\
\text { USD } \$ 724 \\
\text { Custos totais } \\
\text { USD\$ } 5301\end{array}$ & $\begin{array}{l}\text { Inativo: prática de } \mathrm{AF}=0 \text { minuto/semana } \\
\text { Ativo: prática de } \mathrm{AF} \geq 120 \text { minutos/ } \\
\text { semana }\end{array}$ \\
\hline
\end{tabular}

em decorrência de doenças relacionadas à inatividade física e, destes, aproximadamente USD\$ 154 milhões foram devidos diretamente à DM2, em $2002^{14}$. Estimativas mais recentes realizadas com dados de usuários do sistema de saúde indicaram que, em 2006 e 2007, 46\% dos custos totais de saúde foram atribuídos a doenças relacionadas à dieta inadequada e inatividade física, contabilizando USD $\$ 1,8$ trilhões no tota ${ }^{15}$.

Pesquisadores chineses que analisaram dados do NationalNutritionand Health Survey, estima- ram que os custos de USD $\$ 1,3$ bilhões foram utilizados para o tratamento de hipertensão arterial, de USD \$ 0,7 bilhões com DM2, de USD\$ 1,2 bilhões com doença arterial coronariana e de USD $\$ 2$ bilhões com tratamento de pessoas com infarto do miocárdio, os quais foram atribuídos diretamente à inatividade física ${ }^{16}$.

Os resumos dos principais resultados de cada um dos 24 artigos originais revisados são apresentados no Quadro 3, descrevendo os autores, o país de origem e a população envolvida no estudo. 
Quadro 2. Resumo dos estudos sobre custos diretos com saúde e inatividade física com estimativas populacionais.

\begin{tabular}{|c|c|c|c|c|}
\hline País & Autores & $\begin{array}{l}\text { Ano do } \\
\text { estudo }\end{array}$ & Condição & Consequência \\
\hline Brasil & Bielemann et al. ${ }^{10}$ & 2010 & $\downarrow 50 \%$ de inatividade física & $\begin{array}{l}\downarrow 13 \% \text { do custo com medicamentos para } \\
\text { diabetes e } 12 \% \text { para hipertensão. } \\
\downarrow \text { custo USD } \$ 1,14 \text { bilhões }\end{array}$ \\
\hline Austrália & Cadilhac et al. ${ }^{11}$ & 2011 & $\downarrow 10 \%$ de inatividade física & $\begin{array}{l}\downarrow 14 \% \text { nos custos totais. } \\
\text { USD } \$ 91,5 \text { milhões }\end{array}$ \\
\hline Canadá & Katzmarzyk et al. ${ }^{12}$ & 2000 & $\downarrow 10 \%$ de inatividade física & $\downarrow$ USD\$ 101 milhões por ano \\
\hline Canadá & Janssen $^{13}$ & 2012 & $\begin{array}{l}\text { Prevalência de inatividade } \\
\text { física de } 83 \%\end{array}$ & $\begin{array}{l}\text { Utilização de } 3,7 \% \text { dos custos totais com } \\
\text { saúde }\end{array}$ \\
\hline Inglaterra & Allender et al. ${ }^{14}$ & 2007 & $\begin{array}{l}\text { Mortes por doenças } \\
\text { relacionadas à inatividade } \\
\text { física }\end{array}$ & Custo de USD $\$ 1,62$ bilhões \\
\hline Inglaterra & Scarbourgh et al. ${ }^{15}$ & 2011 & $\begin{array}{l}\text { Inatividade física e dieta } \\
\text { inadequada }\end{array}$ & $\begin{array}{l}46 \% \text { dos custos em saúde } \\
\text { USD\$ } 1,8 \text { bilhões }\end{array}$ \\
\hline China & Zhang e Chaaban ${ }^{16}$ & 2013 & Inatividade física & $\begin{array}{l}\text { Comprometimento de } 15 \% \text { dos recursos } \\
\text { para a saúde }\end{array}$ \\
\hline EUA & Garret et al. ${ }^{17}$ & 2004 & $\begin{array}{l}49 \% \text { da população } \\
\text { insuficientemente ativa }\end{array}$ & Custo de USD\$ 83,6 milhões por ano \\
\hline
\end{tabular}

\section{Estudos de coorte}

Entre os 24 estudos analisados, os de coorte são especialmente importantes por quantificarem os custos relacionados à inatividade física de maneira mais precisa que os transversais, que utilizam de métodos indiretos de análise, como custos autorreferidos e estimativas a partir do risco atribuído à inatividade física. As pesquisas analisadas nesta revisão tiveram o tempo médio de dois anos para o seguimento dos participantes. A mais antiga foi realizada entre os anos de 1995 e 1996, nos Estados Unidos ${ }^{18}$, mostrando que entre 5689 usuários de planos de saúde, os homens utilizam 39\% menos recursos que mulheres e os encargos de saúde foram 4,7\% maiores para indivíduos que não fazem $\mathrm{AF}$, comparados aos que fazem ao menos um dia na semana.

Nos anos seguintes, entre 1996 e 1999, Anderson et al. ${ }^{19}$ também acompanharam uma coorte de usuários de planos de saúde nos Estados Unidos e contabilizaram maiores custos para o gru- po de indivíduos inativos (nenhum dia de AF por semana), comparado ao grupo de fisicamente ativos (4 dias ou mais de AF por semana). Ainda, $23,5 \%$ dos encargos em saúde foram associados à inatividade física e ao excesso de peso.

Resultados obtidos a partir de uma coorte de mulheres nascidas entre 1946 e 1951 na Austrália, mostraram que, as menos ativas (<40 MET-minutos por semana) e com maior tempo sentado ( $\geq 8$ h/dia) gastaram em média USD\$ 92,53 a mais por ano, comparadas ao grupo de mulheres fisicamente ativas ( $\geq 40$ MET-minutos por semana) e com menor tempo sentado por dia $(<8 \mathrm{~h} /$ dia). Além disso, o grupo de mulheres fisicamente inativas, mas com menor tempo dispendido sentado por dia, apresentou o custo anual superior, em USD\$ 109 por ano ${ }^{20}$.

Um estudo conduzido na Itália, com 179 pacientes diabéticos com média de idade 62 anos, propôs intervenção composta de aconselhamento para a prática de AF durante dois anos (por telefone), na qual os participantes foram 
Quadro 3. Resumo descritivo dos 24 estudos analisados, por autor, país, amostra e população de estudo e resultado principal.

\begin{tabular}{|c|c|c|c|}
\hline Autor- ano & País & $\begin{array}{c}\text { Amostra e } \\
\text { população de estudo }\end{array}$ & $\begin{array}{l}\text { Resultado } \\
\text { principal }\end{array}$ \\
\hline $\begin{array}{l}\text { Pronk et al., } \\
1999^{18}\end{array}$ & USA & $\begin{array}{l}5689 \text { pacientes } \\
>40 \text { anos }\end{array}$ & $\begin{array}{l}\text { Encargo de saúde } 4,7 \% \text { maior em indivíduos } \\
\text { sedentários. }\end{array}$ \\
\hline $\begin{array}{l}\text { Pratt et al., } \\
2000^{32}\end{array}$ & USA & $\begin{array}{l}35 \text { mil pessoas } \\
>15 \text { anos. }\end{array}$ & $\begin{array}{l}\text { Inatividade física custou USD } \$ 29,2 \text { bilhões. } \\
\text { Custo anual por pessoa inativa USD } \$ 1412 \text {. }\end{array}$ \\
\hline $\begin{array}{l}\text { Katzmarzyk } \\
\text { et al., } 2000^{12}\end{array}$ & Canadá & Estimativas para a população. & $\begin{array}{l}\text { Custos de USD } \$ 1,4 \text { bilhões devido à inatividade } \\
\text { física. Redução de } 4,7 \% \text { nos custos para cada dia } \\
\text { de atividade física reportada por semana. }\end{array}$ \\
\hline $\begin{array}{l}\text { Perkins e } \\
\text { Clark } 2001^{22}\end{array}$ & USA & $\begin{array}{l}655 \text { pessoas } \\
\geq 55 \text { anos }\end{array}$ & $\begin{array}{l}\text { Custos totais e com serviço de emergência são } \\
\text { menores, quanto maior o tempo de prática de } \\
\text { caminhada. }\end{array}$ \\
\hline $\begin{array}{l}\text { Wang et al., } \\
2004^{26}\end{array}$ & USA & $\begin{array}{l}2472 \text { pessoas } \\
>19 \text { anos }\end{array}$ & $\begin{array}{l}\text { Pessoas inativas gastaram por ano USD } \$ 13 \\
\text { bilhões a mais que ativas. }\end{array}$ \\
\hline $\begin{array}{l}\text { Anderson et } \\
\text { al., } 2005^{19}\end{array}$ & USA & $\begin{array}{l}\text { Dados de } 8 \text { mil usuários de } \\
\text { plano de saúde } \\
>40 \text { anos }\end{array}$ & $\begin{array}{l}\text { Estimados que } 23,5 \% \text { dos custos com saúde } \\
\text { sejam associados ao NAF e excesso de peso. }\end{array}$ \\
\hline $\begin{array}{l}\text { Garret et al., } \\
2004^{17}\end{array}$ & USA & $\begin{array}{l}\text { 1,5 milhões de usuários de } \\
\text { planos de saúde }\end{array}$ & $\begin{array}{l}\text { Estimados USD\$ } 83 \text { milhões de custos devido à } \\
\text { inatividade física. }\end{array}$ \\
\hline $\begin{array}{l}\text { Di Loreto et } \\
\text { al., } 2005^{21}\end{array}$ & Itália & $\begin{array}{l}179 \text { pacientes com DM2 } \\
\text { média de idade } 62 \text { anos }\end{array}$ & $\begin{array}{l}\text { O aumento do gasto energético ao longo de } 2 \\
\text { anos representou redução de custos. Sedentários } \\
\text { tiveram aumento de custo no período estudado. }\end{array}$ \\
\hline $\begin{array}{l}\text { Wang et al., } \\
2005^{27}\end{array}$ & USA & $\begin{array}{l}42520 \text { pessoas } \\
\geq 65 \text { anos }\end{array}$ & $\begin{array}{l}\text { Custos com medicamentos e hospitalização } \\
\text { menores quanto maior o NAF. }\end{array}$ \\
\hline $\begin{array}{l}\text { Bertoldi et al., } \\
2006^{31}\end{array}$ & Brasil & $\begin{array}{l}3182 \text { pessoas } \\
>20 \text { anos }\end{array}$ & $\begin{array}{l}\text { Quanto maior o NAF, menor a utilização e } \\
\text { custos com medicamentos. }\end{array}$ \\
\hline $\begin{array}{l}\text { Allender et } \\
\text { al., } 2007^{14}\end{array}$ & Inglaterra & Estimativas para a população & $\begin{array}{l}\text { Custo com doenças diretamente associadas } \\
\text { à inatividade física estimado em USD } \$ 1,62 \\
\text { bilhões. }\end{array}$ \\
\hline $\begin{array}{l}\text { Brown et al., } \\
2008^{30}\end{array}$ & Austrália & $\begin{array}{l}7004 \text { mulheres } \\
50 \text { a } 55 \text { anos }\end{array}$ & $\begin{array}{l}\text { Custos menores quanto maior o NAF, } \\
\text { principalmente quando associada ao excesso de } \\
\text { peso }\end{array}$ \\
\hline $\begin{array}{l}\text { Liu-Ambrose } \\
\text { et al., } 2008^{28}\end{array}$ & Canadá & $\begin{array}{l}209 \text { pessoas } \\
\geq 65 \text { anos }\end{array}$ & Custo inversamente associado ao NAF. \\
\hline $\begin{array}{l}\text { Woolcott et } \\
\text { al., } 2010^{29}\end{array}$ & Canadá & $\begin{array}{l}24281 \text { pessoas } \\
\geq 65 \text { anos }\end{array}$ & $\begin{array}{l}\text { Quanto maior o gasto energético em AF, menor } \\
\text { o custo com internações e custos totais com } \\
\text { saúde. }\end{array}$ \\
\hline $\begin{array}{l}\text { Bielemann et } \\
\text { al., } 2010^{10}\end{array}$ & Brasil & População de Pelotas-RS & $\begin{array}{l}\text { Estimativa de redução de custos com } \\
\text { internações e medicamentos com aumento do } \\
\text { NAF populacional }\end{array}$ \\
\hline
\end{tabular}




\begin{tabular}{|c|c|c|c|}
\hline \multicolumn{4}{|c|}{ Quadro 3. continuação } \\
\hline Autor- ano & País & $\begin{array}{c}\text { Amostra e } \\
\text { população de estudo }\end{array}$ & $\begin{array}{l}\text { Resultado } \\
\text { principal }\end{array}$ \\
\hline $\begin{array}{l}\text { Scarborough } \\
\text { et al., } 2011^{15}\end{array}$ & Inglaterra & $\begin{array}{l}\text { Usuários do sistema de saúde } \\
\text { inglês. }\end{array}$ & $\begin{array}{l}\text { USD } 1,8 \text { trilhões dos custos anuais atribuídos à } \\
\text { inatividade física. }\end{array}$ \\
\hline $\begin{array}{l}\text { Cadilhac et } \\
\text { al., } 2011^{11}\end{array}$ & Austrália & $\begin{array}{l}\text { Estimativas para toda a } \\
\text { população australiana. }\end{array}$ & $\begin{array}{l}\text { Estimada a economia de } 14 \% \text { nos custos devido } \\
\text { à redução de } 10 \% \text { de inativos na população }\end{array}$ \\
\hline $\begin{array}{l}\text { Yang et al., } \\
2011^{33}\end{array}$ & Japão & $\begin{array}{l}483 \text { pessoas } \\
\text { média de idade } 75 \text { anos }\end{array}$ & $\begin{array}{l}\text { Custos menores para pessoas com melhor } \\
\text { aptidão física. } \\
\text { Quanto maior o NAF, menor o custo. }\end{array}$ \\
\hline $\begin{array}{l}\text { Codogno et } \\
\text { al., } 2011^{24}\end{array}$ & Brasil & $\begin{array}{l}121 \text { pacientes com DM2 média } \\
\text { de idade } 60 \text { anos }\end{array}$ & $\begin{array}{l}\text { Sem diferença nos custos com consultas e } \\
\text { medicamentos para DM2, segundo o NAF. }\end{array}$ \\
\hline $\begin{array}{l}\text { Codogno et } \\
\text { al., } 2011^{25}\end{array}$ & Brasil & $\begin{array}{l}118 \text { pacientes com DM2 idade } \\
\text { média } 60 \text { anos }\end{array}$ & $\begin{array}{l}\text { Associação do NAF com custos com } \\
\text { medicamentos para outras doenças e consultas. }\end{array}$ \\
\hline $\begin{array}{l}\text { Janssen, } \\
2012^{13}\end{array}$ & Canadá & População Canadá & $\begin{array}{l}\text { Custo total da inatividade física de USD } \$ 5,9 \\
\text { bilhões. }\end{array}$ \\
\hline $\begin{array}{l}\text { Codogno et } \\
\text { al., } 2012^{23}\end{array}$ & Brasil & $\begin{array}{l}121 \text { pacientes com DM2 } \\
\geq 50 \text { anos }\end{array}$ & $\begin{array}{l}\text { Sedentários custaram mais que ativos com } \\
\text { despesas com clínico geral e medicamentos para } \\
\text { outras doenças }\end{array}$ \\
\hline $\begin{array}{l}\text { Zhang e } \\
\text { Chaaban, } \\
2013^{16}\end{array}$ & China & Estimativas para a população & $\begin{array}{l}\text { Inatividade física utilizou } 15 \% \text { dos recursos em } \\
\text { saúde e foi responsável pelo custo de USD } \$ 6,7 \\
\text { bilhões em } 2007 \text {. }\end{array}$ \\
\hline $\begin{array}{l}\text { Peeters et al., } \\
2014^{20}\end{array}$ & Austrália & 5535 mulheres de meia idade & $\begin{array}{l}\text { Mulheres fisicamente inativas gastam USD } \$ 158 \\
\text { a mais por ano. }\end{array}$ \\
\hline
\end{tabular}

instruídos a aumentar o NAF com a sua prática em intensidades entre 40 e $60 \%$ da frequência cardíaca de reserva. Após a intervenção, o grupo que apresentou gasto energético (GE) igual a zero MET por hora semanal apresentou aumento dos custos com medicação no período do estudo $(\mathrm{p}<0,01)$. Por outro lado, indivíduos com GE entre 11-20 MET por hora semanal economizaram anualmente USD $\$ 386,00$ por pessoa com medicamentos $(p<0,0001)$, enquanto o grupo com GE > 40 MET economizou USD $\$ 579,00$ por pessoa $^{21}$.

Perkinse Clark ${ }^{22}$ investigaram 655 indivíduos durante 12 meses e observaram que os custos com saúde forammenores, quanto maior o tempo dispendido em atividades físicas $(\mathrm{p}=0,0003)$.

\section{Discussão}

O presenteestudo de revisão teve por objetivo analisar o impacto do NAF sobre os custos com saúde em diferentes países e regiões do mundo. Os resultados mostram que, em todo o mundo, a atividade física é uma variável importante para a economia de recursos financeiros em saúde pública, por estar inversamente associada aos custos com procedimentos de saúde, medicamentos e controle de doenças crônicas.

Convém destacar queos métodos de estimativa do NAF variam muito entre os estudos, assim como os critérios de classificação, o que pode influenciar diferenças nas associações com os custos. $\mathrm{O}$ critério que permite distinguir entre indi- 
víduos fisicamente ativos e inativos proposto pela Organização Mundial de Saúde, de 150 minutos por semana de AF de intensidade moderada ${ }^{34}$, foi empregado em quatro pesquisas ${ }^{13,17,26,32}$. Outros pesquisadores utilizaram o tempo total diário em minutos de atividade como referência, mas adotaram classificações distintas, com quatro divisões de tempo ${ }^{22,31}$. Wang et al. ${ }^{27}$ consideraram apenas a frequência semanal da prática para a classificação do NAF de idosos.

Entre os estudos que analisaram o NAF de acordo com o GE, também houve diferença no método de classificação ${ }^{12,28,29}$. Liu-Ambroseet al. ${ }^{28}$ utilizaram como referencia o GE, em Kcal, como variável contínua para análise de correlação, enquanto Katzmarzyk et al. ${ }^{12}$ estabeleceram o GE em Kj, e o valor de $12,6 \mathrm{~kJ} / \mathrm{kg}$ por diapara distinguir entre ativos e inativos. Por fim, Woolcotet al. ${ }^{29}$ consideraram o GE de $1000 \mathrm{Kcal} / \mathrm{semana}$ em atividades físicas como critério para a mesma distinção.

Dois estudos utilizaram o equivalente metabólico (MET) da atividade como indicador, entretanto, adotando critérios distintos de classificação do $\mathrm{NAF}^{20,21}$, considerando fisicamente ativas as mulheres que empregaram 40 MET-minutoou mais por semana em $\mathrm{AF}^{20}$ e classificando os indivíduos em cinco categorias a partir dos METs alcançados ${ }^{21}$. Apenas um estudo utilizou a medida da aptidão física como indicador de NAF, baseando-se nos resultados de testes de força, velocidade e potência muscular ${ }^{33}$.

Tais diferenças dificultam a comparação entre os estudos, entretanto, independente do método empregado, os custos com saúde foram predominantemente superiores nos grupos de indivíduos com menor NAF ou aptidão física.

Outro ponto importante a ser considerado na análise do NAF é o tipo de intervenção a ser empregado por meio de programas de atividade física, pois estes devem atender aos critérios de custo -benefício. As recomendações internacionais de atividade física ${ }^{34,35}$ são propostas visando à prevenção de doenças e à manutenção da qualidade de vida e da capacidade funcional. Consequentemente, visam atender à expectativa de economia de recursos financeiros, por meio da manutenção da saúde da população.Entretanto, um pequeno número de estudos tem avaliado intervenções em AF na perspectiva desse tipo de análise.

Um aspecto importante a ser ressaltado neste trabalho é que a maioria das análises de custo foi realizada a partir do custo direto relativo às despesas com doenças. Ou seja, custo que pode ser estimado a partir de variáveis com valores mensuráveis e que, portanto, podem ser contabilizados. Entretanto, a prática regular de AF pode proporcionar inúmeros benefícios considerados intangíveis, como os efeitos sobre o bem estar mental e melhorias da qualidade de vida. Portanto, o maior NAF é capaz de promover efeitos imensuráveis sobre a economia da saúde. Assim, ao discutir benefícios econômicos da atividade física e/ou prejuízos financeiros decorrentes do estilo de vida sedentário, estes aspectos devem ser lembrados. Dessa forma, os benefícios da promoção da atividade física populacional podem ser ainda maiores e mais significantes para a saúde pública.

Especificamente no Brasil, ainda são escassos os estudos que investigaram as associações entre custos e inatividade física no âmbito populacional. Dessa forma, são necessárias mais pesquisas que analisem e descrevam estas relações de acordo com diferentes tipos de atividade física e para populações específicas, especialmente idosos. Além disso, os estudos de análise de custo-efetividade da prática de $\mathrm{AF}$ auxiliariam no planejamento para a implantação de programas de sucesso.

Em resumo, a literatura disponível, até o momento, aponta a inatividade física como fator importante no aumento dos gastos com saúde, estudos desta natureza auxiliam diretamente no planejamento econômico de países desenvolvidos e em desenvolvimento, ao apresentar evidências de que a inatividade física tem um papel determinante na economia dos recursos em saúde pública. Justificando o desenvolvimento de estratégias de prevenção de doenças crônicas e, consequentemente, dos custos excessivos com procedimentos de saúde, por meio da promoção da atividade física para a população. 


\section{Colaboradores}

DR Bueno, MFNMarucci, JS Codogno e MA Roedigercontribuíram igualmente para a elaboração do trabalho apresentado à Revista Ciência e Saúde Coletiva.DR Bueno, autora principal, é mentora da ideia da pesquisa, realizou as buscas nos bancos de dados e seleção dos manuscritos adequados, organização dos resultados e redação de todo o manuscrito. MFN Marucci foi responsável pela revisão dos métodos, da redação de todo trabalho e colaborou para discussão dos resultados. JS Codogno colaborou na elaboração dos objetivos e métodos da pesquisa, seleção dos trabalhos adequados e análise dos manuscritos, redação dos resultados e contribuição na discussão. MA Roediger colaborou com a organização dos resultados dos artigos selecionados para a revisão, elaboração das tabelas, revisão, análise e discussão da versão final do manuscrito.

\section{Agradecimentos}

Agradecemos à Coordenação de Aperfeiçoamento de Pessoal de Ensino Superior (CAPES) pelo apoio financeiro que possibilitou a realização deste trabalho.

\section{Referências}

1. Lee I, Shiroma EJ, Lobelo F, Puska P, Blair SN, Katzmarzyk PT, for the Lancet Physical Activity Series Working Group. Effect of physical inactivity on major non-communicable diseases worldwide: An analysis of burden of disease and life expectancy. Lancet 2012; 380(9838):219-229.

2. Oldridge NB. Economic burden of physical inactivity: healthcare costs associated with cardiovascular disease. Eur J Cardiovasc Prev Rehabil 2008; 15(2):130-139.

3. Hex N, Bartlett C, Wright D, Taylor M, Varley D. Estimating the current and future costs of type 1 and type 2 diabetes in the United Kingdom, including direct health costs and indirect societal and productivity cost. Diabet Med 2012; 29(7):855-862.

4. Scottish Executive. Let's make Scotland more active: A strategy for physical activity. Edinburgh: The Stationary Office; 2003.

5. World Health Organization (WHO). Global Health Observatory data (GHO) - Health Financing. [acessado 2015 jan 15]. Disponível em: http://www.who.int/ gho/health_financing/en/

6. Malta DC, Moura EA, Castro AM, Cruz DKA, Neto OLM, Monteiro CA. Padrão de atividades físicas em adultos brasileiros: resultados de um inquérito por entrevistas telefônicas, 2006. Epidemiol Serv Saúde 2009; 18(1):7-16.

7. Warburton DER, Nicol CW, Bredin SSD. Health benefits of physical activity: the evidence. CMAJ 2006; 174(6):801-809.

8. Florindo AA, Hallal PC, Moura EA, Malta DC. Prática de atividades físicas e fatores associados em adultos, Brasil, 2006. Rev Saude Publica 2009; 43(Supl. 2):65-73.

9. Freire RS, Lélis FLO, Fonseca Filho JA, Nepomuceno MO, Silveira MF. Prática regular de atividade física: estudo de base populacional no Norte de Minas Gerais, Brasil. Rev Bras Med Esporte 2014; 20(5):345-349.

10. Bielemann RM, Knuth AG, Hallal PC. Atividade física e redução de custos por doenças crônicas para o Sistema Único de Saúde. Rev Bras Ativ Fís Saúde 2010; 15(1):914.

11. Cadilhac DA, Cumming TB, Sheppard L, Pearce DC, Carter R, Manus A. The economic benefits of reducing physical inactivity: an Australian example. Int $\mathrm{J} \mathrm{Be}$ havNutr Phys Act 2011; 8:99.

12. Katzmarzyk PT, Gledhill N, Shephard RJ. The economic burden of physical inactivity in Canada. CMAJ 2000; 163(11):1435-1440.

13. Janssen I. Health care costs of physical inactivity in Canadian adults. Appl. Physiol. Nutr. Metab 2012; 37(4):803-806.

14. Allender S, Foster C, Scarborough P, Rayner M. The burden of physical activity-related ill health in the UK. J Epidemiol Community Health 2007; 61(4):344-348.

15. Scarborough P, Bhatnagar P, Wickramasinghe KK, Allender S, Foster C, Rayner M. The economic burden of ill health due to diet, physical inactivity, smoking, alcohol and obesity in the UK: an update to 2006-07 NHS costs. J Public Health 2011; 33(4):527-535.

16. Zhang J, Chaaban J. The economic cost of physical inactivity in China.Prev Med 2013; 56: 75-78.

17. Garret NA, Brasure M, Schmitz KH, Schultz MM, Huber MR. physical inactivity: direct cost to a health plan. Am J Prev Med 2004; 27(4):304-309. 
18. Pronk NP, Goodman MJ, O'Connor PJ, Martinson BC. Relationship between modifiable health risks and short-term health care charges. JAMA 1999; 282(23):2235-2239.

19. Anderson LH, Martinson BC, Crain Al, Pronk NP, Whitebird RR, Fine LJ, O'Connor PJ. Health care charges associated with physical inactivity, overweight, and obesity. Prev Chronic Dis 2005: 2(4):A09.

20. Peeters GMEE, Mishra GD, Dobson AJ, Brown WJ. Health Care Costs Associated with Prolonged Sitting and Inactivity. Am J Prev Med 2014; 46(3):265-272.

21. Di Loreto C, Fanelli C, Lucidi P, Murdolo G, De Cicco A, Parlanti N. Make your diabetic patients walk: longterm impact of different amounts of physical activity on type 2 diabetes. Diabetes Care 2005; 28(6):12951302.

22. Perkins JA, Clark DO. Assessing the association of walking with health services use and costs among socioeconomically disadvantaged older adults. Prev Med 2001; 32(6):492-501.

23. Codogno JS, Fernandes RA, Monteiro HL. Prática de atividades físicas e custo do tratamento ambulatorial de diabéticos tipo 2 atendidos em unidade básica de saúde. Arq Bras Endocrinol Metab 2012; 56(1):6-11.

24. Codogno JS, Fernandes RA, Rosa CSC, Bueno DR, Monteiro HL. Custo com tratamento e indicadores de risco em pacientes diabéticos, segundo esquema terapêutico. R. da Educação Física 2011; 22(1):111-118.

25. Codogno JS, Fernandes RA, Sarti FM, Freitas Junior IF, Monteiro HL. The burden of physical activity on type 2 diabetes public healthcare expenditures among adults: a retrospective study. BMC Public Health 2011; 11:275.

26. Wang G, Pratt M, Macera CA, Zheng Z, Heath G. Physical activity, cardiovascular disease, and medical expendituresin U. S. Adults. Ann Behav Med 2004; 28(2):8894.

27. Wang F, McDonald T, Reffit B, Edington DW. BMI, physical activity, and health care utilization/costs among medicare retirees. Obesity Research 2005; 13(8):1450-1457.

28. Liu-Ambrose TYL, Ashe MC, Marra CA. Physical activity and chronic conditions among older adults with multiple chronic conditions, physical activity is independently and inversely associated with health care utilization. BJSM Online 2008; First, published on May 16.
29. Woolcott JC, Ashe MC, Miller WC, Shi P, Marra CA. Does physical activity reduce seniors need for healthcare? A study of 24281 Canadians. Br J Sports Med 2010; 44(12):902-904.

30. Brown WJ, Hockey H, Dobson AJ. Physical activity, Body Mass Index and health care costs in mid-age Australian women. Aust N Z J Public Health 2008; 32:150155.

31. Bertoldi AD, Hallal PC, Barros AJD. Physical activity and medicine use: evidence from a population-based study. BMC Public Health 2006, 6:224.

32. Pratt M, Macera CA, Wang G. Higher direct medical costs associatedwith physical inactivity. Phys Sportsmed 28(10):63-70.

33. Yang G, Niu K, Fugita K, Hozawa A, Ohmori-Matsuda K, Kuriyama S, Nakaya N, Ebihara S, Okazaki T, Guo H, Miura C, Takahashi H, Arai H, Tsuji I, Nagatomi R. Impact of physical activity and performance on medical care costs among the Japanese elderly. Geriatr Gerontol Int 2011; 11(2):157-165.

34. World Health Organization (WHO). Global recommendations on physical activity for health, 2010. [acessado 2015 jan 15]. Disponível em: http://www.who.int/ dietphysicalactivity/factsheet_recommendations/en/ index.html.

35. Haskell WL, Lee IM, Pate RR, Powell KE, Blair SN, Franklin BA, Macera CA, Heath GW, Thompson PD, Bauman A. Physical activity and public health: updated recommendation for adults from the American College of Sports Medicine and the American Heart Association. Med Sci Sports Exerc 2007; 39(8):1423-1434.

Artigo apresentado em 05/03/2015

Aprovado em 16/07/2015

Versão final apresentada em 18/07/2015 